\title{
Design of Adaptive SMC for EOD Robotics Manipulator Arm
}

\author{
Naseem Ahmad, Fahad Mumtaz Malik, Sajjad Hussain, Omair Altaf \\ DEE, CE\&ME, National University of Sciences and technology \\ 44000, Islamabad, Pakistan \\ naseem.ahmad83@ee.ceme.edu.pk; malikfahadmumtaz@yahoo.com; sajjad.hussain83@ee.ceme.edu.pk; \\ omair.altaf83@ee.ceme.edu.pk
}

\begin{abstract}
This paper addresses the use of robust control technique ASMC for nonlinear multiple input multiple output RRRP manipulator arm precisely to be used for EOD robots in the presence of parametric uncertainties and external disturbances. In the proposed scheme for control law, model parameters are assumed unknown and are estimated via adaptive laws. Stability of the proposed controller is proved via Lyapunov theory and tracking of the desired path is guaranteed. Advantage of ASMC over other techniques is, the availability of a priori information about the upper bounds of the uncertainties are not required. Extensive simulations are carried out to show the effectiveness of the proposed controller compared to standard SMC.
\end{abstract}

Keywords: Sliding Mode Control (SMC), Adaptive Sliding Mode Control (ASMC), Explosive Ordinance Disposal (EOD), Mobile Robots.

\section{Introduction}

Explosive Ordnance Disposal (EOD) robot is a mobile robot, used as an alternate for human bomb disposer, in order to carry out detection, identification, rendering and disposing of unexploded explosive material during EOD operations to minimize the risk of human life [1]. The basic operational unit of EOD robot is manipulator arm, which precisely locates the end effector to the desired position. This paper proposes RRRP mechanism, which comprises of three revolute and one prismatic joint at its forearm, making it a four DOF robotic manipulator. The advantage of RRRP manipulator over other manipulators is the addition of prismatic joint at manipulator's forearm that serves the purpose of maximising the versatility of manipulator while working EOD operations in narrow places where there is no sufficient space available for the rotation of links. Practically the manipulator systems are highly nonlinear, coupled, time variant and complex. Manipulator also contains parametric uncertainties and external disturbances. Robust techniques are required to withstand these uncertainties and disturbances.

A number of techniques have been introduced in the literature for the control of manipulators. Most of these techniques are based on linearizing manipulator dynamic models. Linearized model control techniques like PD, PID and Feedback linearization discussed in [2, 3 and 4], $\mathrm{H}_{\infty}$ control and linear parameter varying technique (LQR/LQG) have been discussed in [5]. Stability and performance of these control techniques can be assured by the analytical framework however neglecting model parametric uncertainties and external disturbances lead to degradation in performance of these controllers. A number of nonlinear controllers have also been proposed by researchers, such as dynamic inversion model, which replaces the system's original dynamics by user-defined dynamics, and ensures stability and performance by removing the system nonlinearities; however a major drawback of this technique is that during cancelation the information regarding the nonlinearities of system is lost so the robustness of the controller is degraded as described in [6]. Back stepping is another nonlinear control technique described in [7], which deals with the parametric uncertainties of the system by doing online parameters estimation, a major drawback of this method is the computation of regression matrix also it only guarantees ultimate global asymptotic stability when the disturbance is not varying with time.

SMC and ASMC are the nonlinear robust control techniques to tackle with the above problems. SMC is a control technique where we need to drive the error to the sliding surface after which the system is in sliding mode and is not affected by external disturbance or model parametric uncertainty, a well-known problem with ideal SMC is that of chattering [8]. A well-established method to solve the problem of chattering is to use SMC with signum function replaced by saturation function [9]. In SMC sliding surface is designed that models the desired performance in state space, followed 
by controller design comprising of two parts, equivalent controller and sliding controller. Equivalent controller ensures system state trajectories move to sliding surface and sliding controller is designed to keep the state trajectories on that surface for all future time. Previously SMC and ASMC were proposed for nonlinear systems but the approaches were applied only to simple nonlinear systems [10,11] or the upper bounds were assumed to be known [12].

In this paper ASMC is proposed as robust technique to control a nonlinear multiple inputs and multiple outputs EOD RRRP robotic manipulator system with uncertainties both in system dynamics and its input distribution gain. ASMC is the extension of SMC that can adaptively adjust the controller gain. The advantage of such approach is its robustness to parameter variations and disturbances $[13,14]$ and it doesn't require any a priori information about upper bounds.

The rest of this paper is organized as follows: mathematical modelling of system is carried out in section 2, followed by design of ASMC in section 3, section 4 contains the simulation results and discussion while the paper ends with the conclusion section.

\section{System Modelling}

Mathematical modelling of robotic manipulators starts with frame assignment to the joints, keeping that in view the frame assignment was carried out for our system as shown in fig. 1.

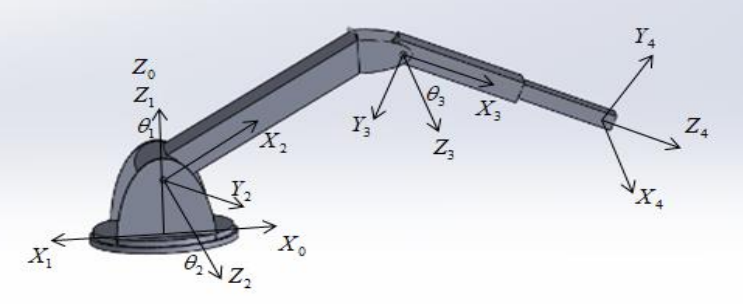

Fig. 1: Frame assignment to RRRP manipulator.

Denavit — Hartenberg (DH) notation parameters are found using method given in [15] shown in table 1.

Table 1: Denavit—Hartenberg parameters for RRRP manipulator.

\begin{tabular}{|c|c|c|c|c|}
\hline Joint & $\boldsymbol{a}_{\boldsymbol{i}-\mathbf{1}}$ & $\boldsymbol{\alpha}_{\boldsymbol{i}-\mathbf{1}}$ & $\boldsymbol{d}_{\boldsymbol{i}}$ & $\boldsymbol{\theta}_{\boldsymbol{i}}$ \\
\hline 1 & 0 & $0^{0}$ & 0 & $\theta_{1}$ \\
\hline 2 & 0 & $90^{0}$ & 0 & $\theta_{2}$ \\
\hline 3 & $l_{2}$ & $0^{0}$ & 0 & $\theta_{3}$ \\
\hline 4 & $l_{3}$ & $90^{0}$ & $d_{4}$ & $0^{0}$ \\
\hline
\end{tabular}

Using this DH parameters table the transformation matrices $\left({ }_{i}^{i-1} T\right)$ between the joints were calculated from those transformation matrices the subsequent rotation matrices $\left({ }_{i}^{i-1} R\right)$ and position vectors $\left({ }_{i}^{i-1} P\right)$ were found using method shown in [15]. Using Newton Euler method and plugging in the previously calculated matrices and vectors the kinematic and dynamic equations for the robotic arm were obtained. Final dynamical equations also known as the torque equations were found and given in appendix A. The mass matrix $M(\ddot{q})$, velocity vector $V(q, \dot{q})$ expressing the Coriolis, centrifugal forces and gravity vector $G(q)$ are extracted from torque equations according to the method given in [15] and generally written as:

$$
\tau=M(q) \ddot{q}+V(q, \dot{q})+G(q)
$$

where $q=\left[\begin{array}{llll}q_{1} & q_{2} & q_{3} & q_{4}\end{array}\right]^{T}, q_{1}=\theta_{1}, q_{2}=\theta_{2}, q_{3}=\theta_{3}$ and $q_{4}=d_{4}$. where $\theta$ is joint angle and $d$ is link offset 
Finding acceleration from Eq. (1) gives:

$$
\ddot{q}=M(q)^{-1}(\tau-V(q, \dot{q})-G(q))
$$

Converting into state space, let $x_{1}=q_{1}, x_{2}=\dot{x}_{1}, x_{3}=q_{2}, x_{4}=\dot{x}_{3}, x_{5}=q_{3}, x_{6}=\dot{x}_{5}, x_{7}=q_{4}$ and $x_{8}=\dot{x}_{7}$. System modelling equation containing parametric uncertainties and external disturbances is written as:

$$
\begin{gathered}
\dot{x}=f(x)+\Delta f(x)+(g(x)+\Delta g(x)) u+w \\
y=h(x)
\end{gathered}
$$

where $x=\left[\begin{array}{llllllll}x_{1} & x_{2} & x_{3} & x_{4} & x_{5} & x_{6} & x_{7} & x_{8}\end{array}\right]^{T}$ represents state vector, $y=\left[\begin{array}{llll}x_{1} & x_{3} & x_{5} & x_{7}\end{array}\right]^{T}$ represents output vector, $f(x)$ and $g(x)$ represent nominal parts of the system, $\Delta f(x)$ and $\Delta g(x)$ represent variations and uncertainties in parameters and $w$ represents external disturbances.

\section{Design of Adaptive Sliding Mode Control}

Uncertainties in system dynamics and external disturbances are unknown in practical systems, the upper bounds of uncertainties are very hard to find. Therefore we need a robust technique that does not required a priori information about the upper bounds of system uncertainties. For this purpose we have used adaptive sliding mode control approach which is most robust and can adaptively adjust the controller gain without any a priori information about the parametric uncertainties of system. Adaptive sliding mode controller is comprised of two parts, one deals with nominal system and the other controls system parameter variations and external disturbances. In adaptive SMC first step is defining sliding surface, assumed to be:

$$
s=C^{T} e
$$

where $C=c_{1}, c_{2}, c_{3}, \ldots, c_{n}$ are positive constants and $e=\left[e_{1}, e_{2}, e_{3}, \ldots, e_{n}\right]^{T}$ are errors between actual and desired states, $\mathrm{n}$ is order of the system. $c_{1}, c_{2}, c_{3}, \ldots, c_{n}$ are selected ensuring the polynomial $c_{n} \gamma^{n-1}+c_{n-1} \gamma^{n-2}+\cdots+c_{1}$ must be Hurwitz. Decaying rate of tracking error can be changed by changing value of $c$. Our main goal is to track the desired output states, let us consider the desired trajectories for RRRP manipulator are:

$$
x_{d}=\left[\begin{array}{llll}
x_{d 1} & x_{d 2} & x_{d 3} & x_{d 4}
\end{array}\right]^{T}=[\sin (t) \cos (t) \sin (t) \cos (t)]^{T}
$$

Error vector is given as:

$$
e=\left[e_{1} e_{2} e_{3} e_{4} e_{5} e_{6} e_{7} e_{8}\right]^{T}=\left[x_{1}-x_{d 1} \dot{e}_{1} x_{3}-x_{d 2} \dot{e}_{3} x_{5}-x_{d 3} \dot{e}_{5} x_{7}-x_{d 4} \dot{e}_{7}\right]^{T}
$$

Sliding surfaces are:

$$
s=\left[s_{1} s_{2} s_{3} s_{4}\right]^{T}=\left[c_{1} e_{1}+c_{2} e_{2} c_{3} e_{3}+c_{4} e_{4} c_{5} e_{5}+c_{6} e_{6} c_{7} e_{7}+c_{8} e_{8}\right]^{T}
$$

$C, f(x)$ and $g(x)$ are given as: 


$$
C^{T}=\left[\begin{array}{cccccccc}
c_{1} & c_{2} & 0 & 0 & 0 & 0 & 0 & 0 \\
0 & 0 & c_{3} & c_{4} & 0 & 0 & 0 & 0 \\
0 & 0 & 0 & 0 & c_{5} & c_{6} & 0 & 0 \\
0 & 0 & 0 & 0 & 0 & 0 & c_{7} & c_{8}
\end{array}\right], \quad\left[\begin{array}{l}
x_{2} \\
f_{1}(x) \\
x_{4} \\
f_{2}(x) \\
x_{6} \\
f_{3}(x) \\
x_{8} \\
f_{4}(x)
\end{array}\right], g(x)=\left[\begin{array}{lllll}
0 & 0 & 0 & 0 & \\
g_{11} & g_{12} & g_{13} & g_{14} \\
0 & 0 & 0 & 0 & \\
g_{21} & g_{22} & g_{23} & g_{24} \\
0 & 0 & 0 & 0 & \\
g_{31} & g_{32} & g_{33} & g_{34} \\
0 & 0 & 0 & 0 & \\
g_{41} & g_{42} & g_{43} & g_{44}
\end{array}\right]
$$

To fulfil our objective for tracking the desired trajectories on Lyapunov base stability let the controller be:

$$
\begin{gathered}
u=u_{n o}+u_{s} \\
u_{n o}=-\left(C^{T} g(x)\right)^{-1}\left(C^{T} f(x)-C^{T} \dot{x}_{d}\right) \\
u_{s}=-\left(C^{T} g(x)\right)^{-1} \widehat{\emptyset} \operatorname{sgn}(s)
\end{gathered}
$$

$u_{n o}$ deals with the nominal part of system and $u_{s}$ is the adaptive term. To reduce the chattering phenomenon we replace $\operatorname{sgn}(s)$ by $\operatorname{sat}(s / \epsilon)$, where $\epsilon$ is a thin boundary layer that (8) become:

$$
u_{s}=-\left(C^{T} g(x)\right)^{-1} \widehat{\varnothing} \operatorname{sat}(s / \epsilon)
$$

where $\widehat{\emptyset}$ is the adjustable gain, for which adaptation law is given as:

$$
\dot{\bar{\emptyset}}=\frac{1}{\sigma}\|s\|
$$

The adaptation gain $\sigma$ can tune the speed of $\widehat{\varnothing}$ that must be greater than zero. To confirm the control law let consider a positive number $\emptyset_{d}$ must fulfil $\emptyset_{d}>|E(x, u)|$ for terminal solution $u_{s}=-\emptyset_{d}\left(C^{T} g(x)\right)^{-1} s g n(s)$. Considering adaptation error $\widetilde{\emptyset}=\widehat{\emptyset}-\emptyset_{d}$, lumped uncertainties $|E(x, u)|=C^{T} \Delta g(x)+C^{T} \Delta f(x) u+C^{T} w$ and Lyapunov candidate function:

$$
\begin{gathered}
V=\frac{1}{2} s^{T} s+\frac{1}{2} \sigma \widetilde{\emptyset}^{2} \\
\dot{V}=s \dot{s}+\sigma \widetilde{\varnothing} \dot{\varnothing} \\
\left.=s\left(C^{T} f(x)-C^{T} \dot{x}_{d}+E(x, u)+C^{T} g(x) u\right)\right)+\sigma\left(\widehat{\varnothing}-\emptyset_{d}\right) \dot{\varnothing}
\end{gathered}
$$

Substituting (8) and (10) in (12) gives:

$$
\begin{gathered}
=s(-\widehat{\varnothing} \operatorname{sgn}(s)+E(x, u))+s\left(\widehat{\varnothing}-\emptyset_{d}\right) \operatorname{sgn}(s) \\
=-\emptyset_{d}|s|+E(x, u) s<0
\end{gathered}
$$


Hence through lyapunov stability the convergence of sliding surface and adaptation error is proved, also tracking is guaranteed because error converges to zero in finite time.

\section{Simulation Results and Discussion}

A set of simulations is carried out on matlab/Simulink for RRRP manipulator arm with the following initiations and physical parameters.

Table 2: Initiations and physical parameters.

\begin{tabular}{|c|c|c|c|}
\hline Articulations & Initial position & Mass $(\mathrm{kg})$ & Length $(\mathrm{m})$ \\
\hline$q_{1}$ & 0 & $\mathrm{X}$ & $\mathrm{X}$ \\
\hline$q_{2}$ & 0 & 5 & 0.5 \\
\hline$q_{3}$ & 0 & 5 & 0.5 \\
\hline$q_{4}$ & 0 & 2 & $\mathrm{X}$ \\
\hline
\end{tabular}

$c_{1}, c_{2}, c_{3}, c_{4}, c_{5}, c_{6}, c_{7}, c_{8}$ are taken $2,4,3,5,6,8,7,9$ respectively, $\epsilon$ is 0.05 and $\sigma$ is 0.2 . Simulation results of SMC and ASMC for RRRP manipulator system containing uncertainties and disturbances are shown in figures 2 to 5.
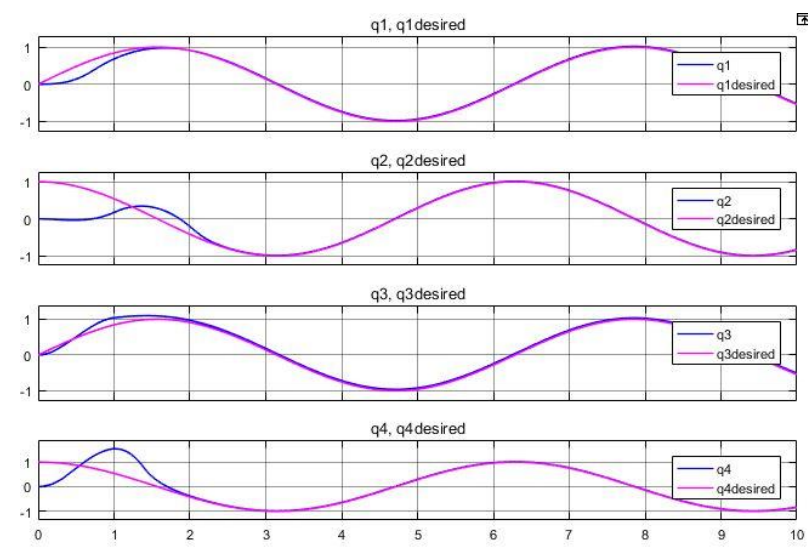

Fig. 2: SMC trajectories tracking.
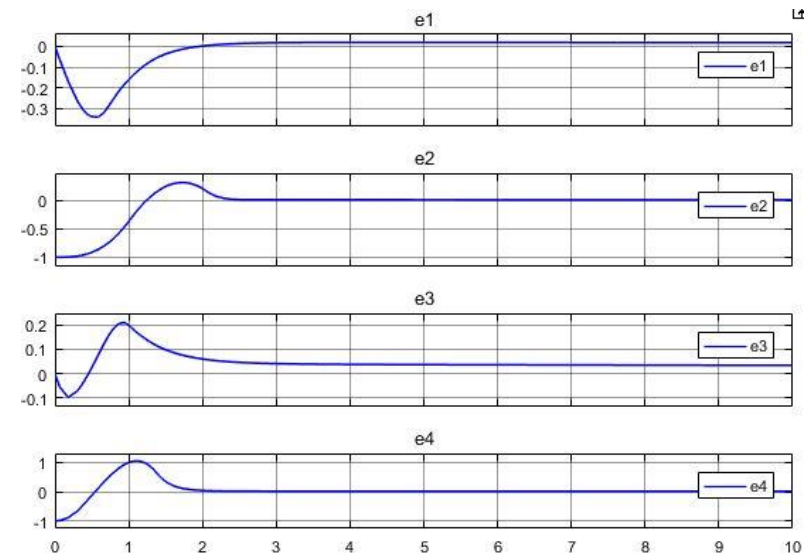

Fig. 3: Errors between actual and desired states for SMC. 

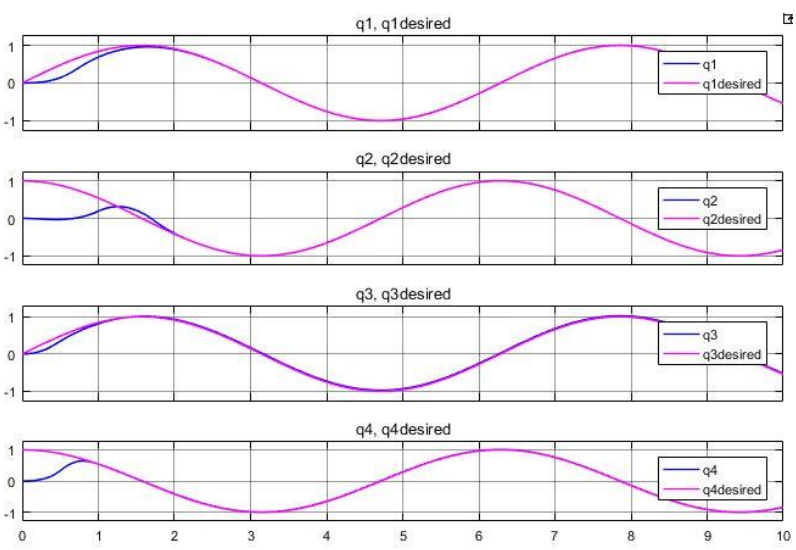

Fig. 4: ASMC trajectories tracking.
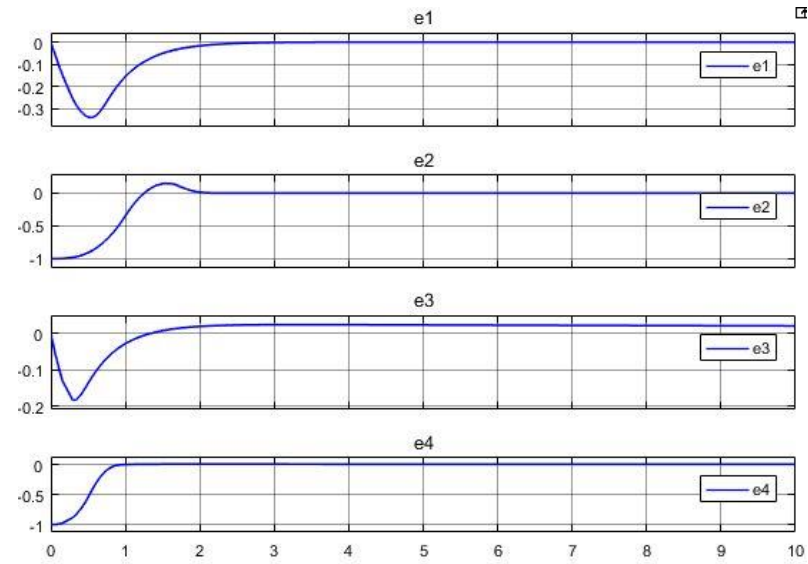

Fig. 5: Errors between actual and desired states for ASMC.

From figures it is clear that the robust control technique, ASMC gives excellent results because of tracking the desired path accurately and quickly with negligible errors. Error plots in figs. 3 and 5 show that the tracking errors converge to zero very rapidly. Both the transient and steady state errors are very low. Absence of chattering in simulation results is due to replacement of signum function with saturation function. Results also show that performance of ASMC is much better than SMC in the presence of unexpected disruptive conditions.

\section{Conclusion}

In this paper robust nonlinear technique ASMC was proposed for nonlinear multiple input multiple output RRRP manipulator arm containing parametric uncertainties and external disturbances. RRRP mechanism was selected due to its better performance in narrow spaces. Upper bounds were also not required for ASMC, which is very hard to find in practical systems. Chattering phenomenon is eliminated by replacing signum function with saturation function. Simulation results proved that the proposed scheme gave excellent performance while withstanding the uncertainties and external disturbances.

\section{References}

[1] L. Aviles, J. Pedraza, E. Gorrostieta and J. Ramos, "Analysis of Dynamic Behavior of an EOD Mechatronic Unit," 2009 Electronics, Robotics and Automotive Mechanics Conference (CERMA), 2009.

[2] K. Valavanis, T. Larsson and S. Gardner, "PD and PID model-based control stability analysis of the PUMA-560 robot manipulator under model mismatch," Journal of Intelligent \& Robotic Systems, vol. 7, no. 2, pp. 233-254, 1993.

[3] I. Akyuz, E. Yolacan, H. Ertunc and Z. Bingul, "PID and state feedback control of a single-link flexible joint robot manipulator", 2011 IEEE International Conference on Mechatronics, 2011. 
[4] I. Akyuz, E. Yolacan, H. Ertunc and Z. Bingul, "PID and state feedback control of a single-link flexible joint robot manipulator," 2011 IEEE International Conference on Mechatronics, 2011.

[5] Z. Doina, "LQG/LQR optimal control for flexible joint manipulator," 2012 International Conference and Exposition on Electrical and Power Engineering, 2012.

[6] H. Evain, M. Rognant, D. Alazard and J. Mignot, "Nonlinear dynamic inversion for redundant systems using the EKF formalism," 2016 American Control Conference (ACC), 2016.

[7] Y. Pan Patel, and U. Ahmad, "Adaptive backstepping control approach for the trajectory tracking of mobile manipulators," 2017 IEEE International Conference on Robotics and Biomimetics (ROBIO), 2017.

[8] M. Ertugrul Celebi, O. Kaynak, and A. Sabanovic, "A comparison of various VSS techniques on the control of automated guided vehicles," Proceedings of the IEEE International Symposium on Industrial Electronics, 1995. ISIE '95., pp. 837-842, 2017.

[9] C. Chen, H. Chiang, and J. Shen, "Fuzzy Sliding Mode Control of a Magnetic Ball Suspension System," International Journal of Fuzzy Systems, vol. 11, no. 2, pp. 97-106, 2009.

[10] J. Baek, M. Jin and S. Han, "A New Adaptive Sliding-Mode Control Scheme for Application to Robot Manipulators," IEEE Transactions on Industrial Electronics, vol. 63, no. 6, pp. 3628-3637, 2016.

[11] A. Nasiri, S. Kiong Nguang and A. Swain, "Adaptive sliding mode control for a class of MIMO nonlinear systems with uncertainties," Journal of the Franklin Institute, vol. 351, no. 4, pp. 2048-2061, 2014.

[12] B. Xiao, S. Yin and O. Kaynak, "Tracking Control of Robotic Manipulators With Uncertain Kinematics and Dynamics," IEEE Transactions on Industrial Electronics, vol. 63, no. 10, pp. 6439-6449, 2016.

[13] V. Utkin, "Sliding Mode inControl and Optimization," Springer-Verlag, Berlin, 1992.

[14] V. Utkin, J. Guldner and J. Shi, "Sliding Modes Control in Electromechanical Systems," CRC press, Boca Raton, 1999.

[15] J. Craig, Introduction to robotics. Upper Saddle River, NJ: Pearson Prentice Hall, 2005.

\section{Appendix A:}

$\tau_{1}=\ddot{\theta}_{1} \mathrm{c}_{2}{ }^{2} 1_{2}{ }^{2} m_{3}+\ddot{\theta}_{1} \mathrm{c}_{2}{ }^{2} 1_{2}{ }^{2} m_{4}+\ddot{\theta}_{1} \mathrm{c}_{2}{ }^{2} c_{3} 1_{2}{ }^{2} m_{3}+\left(\mathrm{c}_{2} d_{4}{ }^{2} \dot{\theta}_{2}{ }^{2} m_{4} \mathrm{~s}_{3}\right) / 2+\left(\mathrm{c}_{3} d_{4}{ }^{2} \dot{\theta}_{2}{ }^{2} m_{4} \mathrm{~s}_{2}\right) / 2+$

$\left(\mathrm{c}_{2} d_{4}{ }^{2} \dot{\theta}_{3}{ }^{2} m_{4} \mathrm{~s}_{3}\right) / 2+\left(\mathrm{c}_{3} d_{4}{ }^{2} \dot{\theta}_{3}{ }^{2} m_{4} \mathrm{~s}_{2}\right) / 2+\mathrm{c}_{1} \mathrm{c}_{2} \mathrm{gl}_{2} m_{3}+\mathrm{c}_{1} \mathrm{c}_{2} \mathrm{gl}_{2} m_{4}-\left(\mathrm{c}_{2} d_{4} \ddot{d}_{4} m_{4} \mathrm{~s}_{3}\right) / 2-$

$\left(\mathrm{c}_{3} d_{4} \ddot{d}_{4} m_{4} \mathrm{~s}_{2}\right) / 2+\left(\ddot{\theta}_{1} \mathrm{c}_{2}{ }^{2} \mathrm{c}_{3}{ }^{2} 1_{2}{ }^{2} m_{3}\right) / 4+\left(\mathrm{c}_{2}{ }^{2} \mathrm{c}_{3}{ }^{2} d_{4}{ }^{2} \dot{\theta}_{1} m_{4}\right) / 4+\ddot{\theta}_{1} \mathrm{c}_{2}{ }^{2} d_{4}{ }^{2} m_{4} \mathrm{~s}_{3}{ }^{2}+$

$\ddot{\theta}_{1} \mathrm{c}_{3}{ }^{2} d_{4}{ }^{2} m_{4} \mathrm{~s}_{2}{ }^{2}+\left(\mathrm{c}_{2}{ }^{2} d_{4}{ }^{2} \dot{\theta}_{1} m_{4} \mathrm{~s}_{3}{ }^{2}\right) / 4+\left(\mathrm{c}_{3}{ }^{2} d_{4}{ }^{2} \dot{\theta}_{1} m_{4} \mathrm{~s}_{2}{ }^{2}\right) / 4+\left(\ddot{\theta}_{1} 1_{2}{ }^{2} m_{3} \mathrm{~s}_{2}{ }^{2} \mathrm{~s}_{3}{ }^{2}\right) / 4+$

$\left(d_{4}{ }^{2} \dot{\theta}_{1} m_{4} \mathrm{~s}_{2}{ }^{2} \mathrm{~s}_{3}{ }^{2}\right) / 4-\left(\ddot{\theta}_{2} \mathrm{c}_{2} \mathrm{c}_{3} d_{4}{ }^{2} m_{4}\right) / 2-\left(\ddot{\theta}_{3} \mathrm{c}_{2} \mathrm{c}_{3} d_{4}{ }^{2} m_{4}\right) / 2+\left(\ddot{\theta}_{2} d_{4}{ }^{2} m_{4} \mathrm{~s}_{2} \mathrm{~s}_{3}\right) / 2+$

$\left(\ddot{\theta}_{3} d_{4}{ }^{2} m_{4} \mathrm{~s}_{2} \mathrm{~s}_{3}\right) / 2+2 \ddot{\theta}_{1} \mathrm{c}_{2}{ }^{2} d_{4} 1_{2} m_{4} \mathrm{~s}_{3}+\left(\ddot{\theta}_{2} \mathrm{c}_{3}{ }^{2} d_{4} 1_{2} m_{4} \mathrm{~s}_{2}\right) / 2+\mathrm{c}_{2} d_{4}{ }^{2} \dot{\theta}_{2} \dot{\theta}_{3} m_{4} \mathrm{~s}_{3}+$

$\mathrm{c}_{3} d_{4}{ }^{2} \dot{\theta}_{2} \dot{\theta}_{3} m_{4} \mathrm{~s}_{2}+2 \mathrm{c}_{2}{ }^{2} \dot{d}_{4} \dot{\theta}_{1} 1_{2} m_{4} \mathrm{~s}_{3}-\ddot{\theta}_{1} \mathrm{c}_{2} 1_{2}{ }^{2} m_{3} \mathrm{~s}_{2} \mathrm{~s}_{3}+\left(\ddot{\theta}_{2} d_{4} 1_{2} m_{4} \mathrm{~s}_{2} \mathrm{~s}_{3}{ }^{2}\right) / 2-$

$2 \mathrm{c}_{2} \dot{\theta}_{1} \dot{\theta}_{2} 1_{2}{ }^{2} m_{3} \mathrm{~s}_{2}-2 \mathrm{c}_{2} \dot{\theta}_{1} \dot{\theta}_{2} 1_{2}{ }^{2} m_{4} \mathrm{~s}_{2}+\left(\mathrm{c}_{2} \mathrm{c}_{3}{ }^{2} d_{4} \dot{\theta}_{2}{ }^{2} 1_{2} m_{4}\right) / 2-\left(\mathrm{c}_{2}{ }^{2} \mathrm{c}_{3}{ }^{2} d_{4} \mathrm{~g} m_{4} \mathrm{~s}_{1}\right) / 2+\ldots+$

$2 \mathrm{c}_{2}{ }^{2} \mathrm{c}_{3} d_{4} \dot{\theta}_{1} \dot{\theta}_{3} 1_{2} \mathrm{~m} 4+2 \ddot{\theta}_{1} \mathrm{c}_{2} \mathrm{c}_{3} d_{4}{ }^{2} m_{4} \mathrm{~s}_{2} \mathrm{~s}_{3}-\left(\ddot{\theta}_{1} \mathrm{c}_{2} \mathrm{c}_{3} 1_{2}{ }^{2} m_{3} \mathrm{~s}_{2} \mathrm{~s}_{3}\right) / 2-2 \mathrm{c}_{2} \mathrm{c}_{3} \dot{\theta}_{1} \dot{\theta}_{2} 1_{2}{ }^{2} m_{3} \mathrm{~s}_{2}-$

$\mathrm{c}_{2} \mathrm{c}_{3} \dot{\theta}_{1} \dot{\theta}_{3} 1_{2}{ }^{2} m_{3} \mathrm{~s}_{2}-2 \mathrm{c}_{3} d_{4} \dot{\theta}_{1} \dot{\theta}_{2} 1_{2} m_{4} \mathrm{~s}_{2}{ }^{2}+2 \mathrm{c}_{2} \mathrm{c}_{3}{ }^{2} d_{4}{ }^{2} \dot{\theta}_{1} \dot{\theta}_{2} m_{4} \mathrm{~s}_{2}+2 \mathrm{c}_{2} \mathrm{c}_{3}{ }^{2} d_{4}{ }^{2} \dot{\theta}_{1} \dot{\theta}_{3} m_{4} \mathrm{~s}_{2}+$

$2 \mathrm{c}_{2}{ }^{2} \mathrm{c}_{3} d_{4}{ }^{2} \dot{\theta}_{1} \dot{\theta}_{2} m_{4} \mathrm{~s}_{3}+2 \mathrm{c}_{2}{ }^{2} \mathrm{c}_{3} d_{4}{ }^{2} \dot{\theta}_{1} \dot{\theta}_{3} m_{4} \mathrm{~s}_{3}+4 \mathrm{c}_{2} \mathrm{c}_{3} d_{4} \dot{d}_{4} \dot{\theta}_{1} m_{4} \mathrm{~s}_{2} \mathrm{~s}_{3}-4 \mathrm{c}_{2} d_{4} \dot{\theta}_{1} \dot{\theta}_{2} 1_{2} m_{4} \mathrm{~s}_{2} \mathrm{~s}_{3}-$

$2 \mathrm{c}_{2} d_{4} \dot{\theta}_{1} \dot{\theta}_{3} 1_{2} m_{4} \mathrm{~s}_{2} \mathrm{~s}_{3}$ 


$$
\begin{aligned}
& \tau_{2}=\ddot{\theta}_{2} d_{4}{ }^{2} m_{4}+\ddot{\theta}_{3} d_{4}{ }^{2} m_{4}+\left(\ddot{\theta}_{2} 1_{2}{ }^{2} m_{3}\right) / 4+\left(\ddot{\theta}_{3} 1_{2}{ }^{2} m_{3}\right) / 4+\ddot{\theta}_{2} \mathrm{c}_{3} 1_{2}{ }^{2} m_{3}+ \\
& \left(\ddot{\theta}_{3} \mathrm{c}_{3} 1_{2}{ }^{2} m_{3}\right) / 2+\ddot{\theta}_{2} \mathrm{c}_{3}{ }^{2} 1_{2}{ }^{2} m_{3}+\ddot{\theta}_{2} \mathrm{c}_{3}{ }^{2} 1_{2}{ }^{2} m_{4}+\ddot{\theta}_{2} 1_{2}{ }^{2} m_{3} \mathrm{~s}_{3}{ }^{2}+ \\
& \ddot{\theta}_{2} 1_{2}{ }^{2} m_{4} \mathrm{~s}_{3}{ }^{2}-\left(\dot{\theta}_{3}{ }^{2} \mathrm{l}_{2}{ }^{2} m_{3} \mathrm{~s}_{3}\right) / 2+2 d_{4} \dot{d}_{4} \dot{\theta}_{2} m_{4}+2 d_{4} \dot{d}_{4} \dot{\theta}_{3} m_{4}- \\
& \mathrm{c}_{3} \ddot{d}_{4} 1_{2} m_{4}-\left(\dot{\theta}_{1}{ }^{2} 1_{2}{ }^{2} m_{3} \mathrm{~s}_{2}{ }^{2} \mathrm{~s}_{3}{ }^{3}\right) / 2+2 \ddot{\theta}_{2} \dot{d}_{4} 2 m_{4} \mathrm{~s}_{3}+\ddot{\theta}_{3} \dot{d}_{4} 2 m_{4} \mathrm{~s}_{3}+ \\
& 2 \dot{d}_{4} \dot{\theta}_{2} 1_{2} m_{4} \mathrm{~s}_{3}+2 \dot{d}_{4} \dot{\theta}_{3} 1_{2} m_{4} \mathrm{~s}_{3}+\left(\mathrm{c}_{2}{ }^{2} \dot{\theta}_{1}{ }^{2} 1_{2}{ }^{2} m_{3} \mathrm{~s}_{3}\right) / 2-\left(\mathrm{c}_{2} \mathrm{c}_{3} d_{4}{ }^{2} \dot{\theta}_{1} m_{4}\right) / 2+ \\
& \mathrm{c}_{3} d_{4} \dot{\theta}_{3}{ }^{2} 1_{2} m_{4}-\dot{\theta}_{2} \dot{\theta}_{3} 1_{2}{ }^{2} m_{3} \mathrm{~s}_{3}+\left(d_{4}{ }^{2} \dot{\theta}_{1} m_{4} \mathrm{~s}_{2} \mathrm{~s}_{3}\right) / 2+\ldots+\left(5 \mathrm{c}_{2} \mathrm{c}_{3}{ }^{2} \dot{\theta}_{1}{ }^{2} 1_{2}{ }^{2} m_{3} \mathrm{~s}_{2}\right) / 4+ \\
& \mathrm{c}_{2} \mathrm{c}_{3}{ }^{2} \dot{\theta}_{1}{ }^{2} 1_{2}{ }^{2} m_{4} \mathrm{~s}_{2}+\left(\mathrm{c}_{2} \mathrm{c}_{3}{ }^{3} \dot{\theta}_{1}{ }^{2} 1_{2}{ }^{2} m_{3} \mathrm{~s}_{2}\right) / 2+\left(\mathrm{c}_{2}{ }^{2} \mathrm{c}_{3} \dot{\theta}_{1}{ }^{2} 1_{2}{ }^{2} m_{3} \mathrm{~s}_{3}\right) / 4+\mathrm{c}_{3}{ }^{3} d_{4} \dot{\theta}_{1}{ }^{2} 1_{2} m_{4} \mathrm{~s}_{2}{ }^{2}+ \\
& \mathrm{c}_{2} d_{4} \dot{\theta}_{1}{ }^{2} l_{2} m_{4} \mathrm{~s}_{2} \mathrm{~s}_{3}{ }^{3}+\left(\mathrm{c}_{2} \mathrm{c}_{3} \dot{\theta}_{1}{ }^{2} 1_{2}{ }^{2} m_{3} \mathrm{~s}_{2} \mathrm{~s}_{3}{ }^{2}\right) / 2+\mathrm{c}_{3} d_{4} \dot{\theta}_{1}^{2} 12 m_{4} \mathrm{~s}_{2}{ }^{2} \mathrm{~s}_{3}{ }^{2}- \\
& \left(\mathrm{c}_{2} \mathrm{c}_{3}{ }^{2} d_{4} \dot{\theta}_{1} \dot{\theta}_{2} 1_{2} m_{4}\right) / 2-\left(\mathrm{c}_{2} \mathrm{c}_{3}{ }^{2} d_{4} \dot{\theta}_{1} \dot{\theta}_{3} 1_{2} m_{4}\right) / 2-\left(\mathrm{c}_{2} d_{4} \dot{\theta}_{1} \dot{\theta}_{2} 1_{2} m_{4} \mathrm{~s}_{3}{ }^{2}\right) / 2- \\
& \left(\mathrm{c}_{2} d_{4} \dot{\theta}_{1} \dot{\theta}_{3} 1_{2} m_{4} \mathrm{~s}_{3}{ }^{2}\right) / 2+\mathrm{c}_{2} d_{4} \dot{\theta}_{1}{ }_{1} 1_{2} m_{4} \mathrm{~s}_{2} \mathrm{~s}_{3}+\mathrm{c}_{2} \mathrm{c}_{3}{ }^{2} d_{4} \dot{\theta}_{1}{ }^{2} 1_{2} m_{4} \mathrm{~s}_{2} \mathrm{~s}_{3} \\
& \tau_{3}=\ddot{\theta}_{2} d_{4}{ }^{2} m_{4}+\ddot{\theta}_{3} d_{4}{ }^{2} m_{4}+\left(\ddot{\theta}_{2} 1_{2}{ }^{2} m_{3}\right) / 4+\left(\ddot{\theta}_{3} 1_{2}{ }^{2} m_{3}\right) / 4+ \\
& \left(\ddot{\theta}_{2} \mathrm{c}_{3} 1_{2}{ }^{2} m_{3}\right) / 2+\left(\dot{\theta}_{2}{ }^{2} \mathrm{l}_{2}{ }^{2} m_{3} \mathrm{~s}_{3}\right) / 2+2 d_{4} \dot{d}_{4} \dot{\theta}_{2} m_{4}+ \\
& 2 d_{4} \dot{d}_{4} \dot{\theta}_{3} m_{4}+\ddot{\theta}_{2} d_{4} 1_{2} m_{4} \mathrm{~s}_{3}+\left(\mathrm{c}_{2}{ }^{2} \dot{\theta}_{1}^{2} 1_{2}{ }^{2} m_{3} \mathrm{~s}_{3}\right) / 2- \\
& \left(\mathrm{c}_{2} \mathrm{c}_{3} d_{4}{ }^{2} \dot{\theta}_{1} m_{4}\right) / 2-\mathrm{c}_{3} d_{4} \dot{\theta}_{2}{ }^{2} l_{2} m_{4}+\left(d_{4}{ }^{2} \dot{\theta}_{1} m_{4} \mathrm{~s}_{2} \mathrm{~s}_{3}\right) / 2+\ldots+\left(\mathrm{c}_{2} \mathrm{c}_{3} \dot{\theta}_{1}{ }^{2} 1_{2}{ }^{2} m_{3} \mathrm{~s}_{2}\right) / 2+ \\
& \mathrm{c}_{2} \mathrm{c}_{3} d_{4} \mathrm{~g} m_{4} \mathrm{~s}_{1}-\left(\mathrm{c}_{2} \mathrm{gl}_{2} m_{3} \mathrm{~s}_{1} \mathrm{~s}_{3}\right) / 2-\left(\mathrm{c}_{3} \mathrm{gl}{ }_{2} m_{3} \mathrm{~s}_{1} \mathrm{~s}_{2}\right) / 2-d_{4} \mathrm{~g} m_{4} \mathrm{~s}_{1} \mathrm{~s}_{2} \mathrm{~s}_{3}- \\
& \mathrm{c}_{2} \mathrm{c}_{3}{ }^{2} d_{4}{ }^{2} \dot{\theta}_{1}^{2} m_{4} \mathrm{~s}_{2}-\mathrm{c}_{2}{ }^{2} \mathrm{c}_{3} d_{4}{ }^{2} \dot{\theta}_{1}^{2} m_{4} \mathrm{~s}_{3}+\left(\mathrm{c}_{2} \mathrm{c}_{3}{ }^{2} \dot{\theta}_{1}{ }^{2} \mathrm{l}_{2}{ }^{2} m_{3} \mathrm{~s}_{2}\right) / 4+ \\
& \left(\mathrm{c}_{2}{ }^{2} \mathrm{c}_{3} \dot{\theta}_{1}^{2} \mathrm{l}_{2}^{2} m_{3} \mathrm{~s}_{3}\right) / 4+\mathrm{c}_{2} d_{4} \dot{\theta}_{1}^{2} \mathrm{l}_{2} m_{4} \mathrm{~s}_{2} \mathrm{~s}_{3} \\
& \tau_{4}=-d_{4} m_{4} \mathrm{c}_{2}^{2} \dot{\theta}_{1}^{2} \mathrm{~s}_{3}{ }^{2}-1_{2} m_{4} \mathrm{c}_{2}{ }^{2} \dot{\theta}_{1}^{2} \mathrm{~s}_{3}-2 d_{4} m_{4} \mathrm{c}_{2} \mathrm{c}_{3} \dot{\theta}_{1}^{2} \mathrm{~s}_{2} \mathrm{~s}_{3}- \\
& 1_{2} m_{4} \mathrm{c}_{2} \mathrm{c}_{3} \dot{\theta}_{1}^{2} \mathrm{~s}_{2}+\ldots+\left(d_{4} m_{4} \mathrm{c}_{2} \mathrm{c}_{3} \dot{\theta}_{1} \dot{\theta}_{3}\right) / 2- \\
& \left(d_{4} m_{4} \mathrm{c}_{2} \dot{\theta}_{1} \mathrm{~s}_{3}\right) / 2+\mathrm{g} m_{4} \mathrm{~s}_{1} \mathrm{c}_{2} \mathrm{~s}_{3}-d_{4} m_{4} \mathrm{c}_{3}{ }^{2} \dot{\theta}_{1}{ }^{2} \mathrm{~s}_{2}{ }^{2}-\left(d_{4} m_{4} \mathrm{c}_{3} \dot{\theta}_{1} \mathrm{~s}_{2}\right) / 2 \\
& +\mathrm{g} m_{4} \mathrm{~s}_{1} \mathrm{c}_{3} \mathrm{~s}_{2}-\ddot{\theta}_{2} 1_{2} m_{4} \mathrm{c}_{3}-\left(d_{4} m_{4} \dot{\theta}_{1} \dot{\theta}_{2} \mathrm{~s}_{2} \mathrm{~s}_{3}\right) / 2-\left(d_{4} m_{4} \dot{\theta}_{1} \dot{\theta}_{3} \mathrm{~s}_{2} \mathrm{~s}_{3}\right) / 2- \\
& 1_{2} m_{4} \dot{\theta}_{2}^{2} \mathrm{~s}_{3}-d_{4} m_{4} \dot{\theta}_{2}^{2}-2 d_{4} m_{4} \dot{\theta}_{2} \dot{\theta}_{3}-d_{4} m_{4} \dot{\theta}_{3}^{2}+\ddot{d}_{4} m_{4}
\end{aligned}
$$

